\title{
The Stick-Slip Vibration and Bifurcation of a Vibro-Impact System with Dry Friction
}

\author{
Quanfu Gao and Xingxiao Cao*
}

School of Mechanical Engineering, Lanzhou Jiaotong University, Lanzhou, 730070, China

\begin{abstract}
In this paper, the periodic motion, bifurcation and chatter of two-degree-of-freedom vibratory system with dry friction and clearance were studied. Slip-stick motion and the impact of system motions were analyzed and numerical simulations were also carried out. The results showed that the system possesses rich dynamics characterized by periodic motion, stick-slip-impact motion, quasi-periodic motion and chaotic attractors, and the routs from periodic motions to chaos observed via Hof bifurcation or period-doubling bifurcation. Furthermore, it was found that there exists the chatter phenomena induced by dry friction in low frequency, and the windows of chaotic motion are broadened in the area of higher excitation frequencies as the dry friction increases.
\end{abstract}

Keywords: Chatter, Dry friction, Hopf bifurcation, Stick-slip, Vibro-Impact.

\section{INTRODUCTION}

The vibro-impact systems with dry friction have been a focus of researchers for a long time, as they are frequently used in everyday life as well as in the engineering systems such as rolling railway wheelset, gear transmission, and disc brake system. In many applications, dry friction, clearance and impact factors often result in sudden change of the vector fields describing dynamic behaviors of mechanical systems [1]. Researches on the impact oscillator with dry friction have important significance in the optimization design of machinery with clearance, noise suppression and reliability. In the past several years, there exists a wide range of research devoted to the analysis of non-smooth systems with dry friction. Feeny and Moon [2] investigated the geometry of chaotic attractors for dry friction oscillators experimentally and numerically, using three different friction laws. The work of Galvanetto $[3,4]$ dealt with dynamics of a three blocks stick-slip system, as well as a one-dimensional map introduced for studying bifurcations in the four dimensional system. In the papers of Awrejcewicz and his collaborators [5-7], the Melnikov-Gruendler approach was used to analyze stick-slip chaos in one-degree-of-freedom and two-degree-of-freedom systems with friction. A chaotic threshold was obtained for both smooth and stick-slip chaotic behaviors in these system. Thomsen and Fidlin [8] considered friction -induced vibrations of a mass-on-belt system, and obtained approximate analytical expressions for the amplitudes and base frequencies of stick-slip and pureslip oscillations.

It is important to note that many studies of dry friction system have been carried out, but few have considered impact oscillators with dry friction. Motion of the impactdry-friction pair of bodies was analyzed by Peterka [9], with

*Address correspondence to this author at the 88 Anning West Road, Lanzhou, Postcard: 730070, China; Tel: 13659437212;

E-mail: cxx2004@126.com emphasis on the influence of dry friction on the system's response. He also performed several experimental tests to verify his solutions. Cone et al. [10] investigated pitchfork bifurcation, grazing bifurcation and Stick-slip vibration of an impact oscillator with addition of dry friction. In fact, the vibro-impact systems coupled with dry friction are closer to the actual applications. In this paper, the focus is on examining stick-slip vibration and bifurcations of twodegree-of-freedom vibro-impact system with dry friction. The influence of dry friction on dynamics of the vibroimpact system with dry friction is elucidated accordingly.

\section{SYSTEM DESCRIPTION}

Two-degree-of-freedom vibro-impact system with dry friction, which was investigated, as shown in Fig. (1), is composed of two mass blocks. The block M1 is connected to the block $\mathrm{M}_{2}$ via a linear spring of stiffness $\mathrm{K}_{1}$ and a damper of viscous damping coefficient $\mathrm{C}_{1}$. The block M2 is connected to a fixed support by a linear spring of stiffness $\mathrm{K}_{2}$ and a damper of viscous damping coefficient $\mathrm{C}_{2}$.

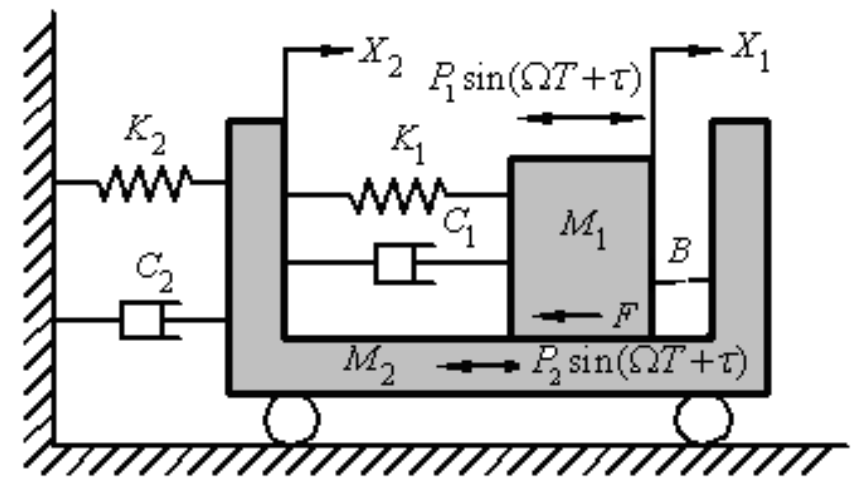

Fig. (1). Model of two-degree-of-freedom vibro-impact system with dry friction. 
Dry friction was considered to be exerted on the contact surface of two blocks when there exists relative motion or a tendency towards relative motion. The dry friction follows Coulomb's friction law. Both masses are subjected to harmonic excitations, namely $\mathrm{P}_{\mathrm{i}} \sin (\Omega \mathrm{T}+\tau),(\mathrm{i}=1,2)$, where $\mathrm{P}_{\mathrm{i}}$ and $\Omega$ are the excitations' strength and frequency respectively. Displacements of the masses M1and M2 are represented by $X_{1}$ and $X_{2}$, respectively, and the clearance between the two masses is denoted by B. Since the block M1 makes a contact with the block $\mathrm{M}_{2}$ with friction, the block $\mathrm{M}_{1}$ can move along, or rest on the surface of block $\mathrm{M}_{2}$. The block $M_{1}$ impacts mutually with the block $M_{2}$ when $X_{1}(T)$ $\mathrm{X}_{2}(\mathrm{~T})=\mathrm{B}$. The impact is described by the conservation law of momentum and a coefficient of restitution $r$, and it is assumed that the duration of impact is negligible compared to the period of the force.

The motion of the system, between consecutive impacts occurring at the stop, was considered. Between any two consecutive impacts, two types of steady motion were observed in the system: one was the sliding motion in which the mass $M_{1}$ slips on the mass $M_{2}$ undersurface, and the velocity of two masses is unequal. While for the slip mode, it is required to observe if the velocity of two masses is equivalent after one time step. If the time point of the velocity of the mass $M_{1}$ is equal to the mass $M_{2}$, the system will enter the other sticking motion if the following relations are true:

$\left|\dot{x}_{1}-\dot{x}_{2}\right|=0,\left|f_{0}\right| \leq f_{s}$

Where $f_{0}$ is the static friction force acting on the mass $\mathrm{M}_{1}$ and $f_{\mathrm{s}}$ is the maximum static friction. In other words, if $\left|f_{0}\right|>f_{s}$, the mass $\mathrm{M}_{1}$ still slips on the mass $\mathrm{M}_{2}$ undersurface and the friction is reversed.

Between two consecutive impacts, the non-dimensional differential equations of sliding motion of the system are given by

$$
\begin{aligned}
& {\left[\begin{array}{cc}
1 & 0 \\
0 & \mu_{m}
\end{array}\right]\left\{\begin{array}{l}
\ddot{x}_{1} \\
\ddot{x}_{2}
\end{array}\right\}+\left[\begin{array}{cc}
2 \xi & -2 \xi \\
-2 \xi & 2 \xi\left(1+\mu_{c}\right)
\end{array}\right]\left\{\begin{array}{l}
\dot{x}_{1} \\
\dot{x}_{2}
\end{array}\right\}+\left[\begin{array}{cc}
1 & -1 \\
-1 & 1+\mu_{k}
\end{array}\right]\left\{\begin{array}{l}
x_{1} \\
x_{2}
\end{array}\right\}} \\
& =\left\{\begin{array}{l}
1-f_{20} \\
f_{20}
\end{array}\right\} \sin (\omega t+\tau)+\left[\begin{array}{l}
-f_{k} \\
f_{k}
\end{array}\right] \operatorname{sgn}\left(\dot{x}_{1}-\dot{x}_{2}\right) \quad\left(x_{1}-x_{2}<b\right) \\
& \text { Where } \operatorname{sgn}(y)= \begin{cases}=1 & (y>0) \\
\in(-1,1) & (y=0) \\
=-1 & (y<0)\end{cases}
\end{aligned}
$$

A dot (.) denotes differentiation with respect to the nondimensional time $t$, and the non-dimensional quantities

$$
\begin{aligned}
& \mu_{m}=\frac{M_{2}}{M_{1}}, \mu_{k}=\frac{K_{2}}{K_{1}}, \mu_{c}=\frac{C_{2}}{C_{1}}, \xi=\frac{C_{1}}{2 \sqrt{K_{1} M_{1}}}, \\
& x_{i}=\frac{X_{i}}{P_{1}+P_{2}}(i=1,2), f_{20}=\frac{P_{2}}{P_{1}+P_{2}}, f_{k}=\frac{F}{P_{1}+P_{2}},
\end{aligned}
$$

$\omega=\Omega \sqrt{\frac{M_{1}}{K_{1}}}, t=T \sqrt{\frac{K_{1}}{M_{1}}}, b=\frac{B K_{1}}{P_{1}+P_{2}}$

have been introduced.

When sticking occurs, it is assumed the maximum static friction has a set value so that $\left|f_{0}\right| \leq f_{s}$. Their accelerations approach to be equivalent and the two-degree-of-freedom vibratory system becomes a single-degree-of-freedom oscillator with the mass $\left(M_{1}+M_{2}\right)$. The change from free motion of both masses to their sticking represents a reduction in the degree of freedom of the system from 2 to 1. The non-dimensional differential equations of stick motion of the system are given by:

$\left(1+\mu_{m}\right) \ddot{x}_{2}+2 \xi \mu_{c} \dot{x}_{2}+\mu_{k} x_{2}=\sin (\omega t+\tau)$

According to $\dot{x}_{1}=\dot{x}_{2}, \ddot{x}_{1}=\ddot{x}_{2}$ and Eq.(3), the static friction force acting on the mass $\mathrm{M}_{1}$ is obtained as follow:

$f_{0}=\frac{\left[\mu_{m}\left(1-f_{20}\right)-f_{20}\right] \sin (\omega t+\tau)+2 \zeta \mu_{c} \dot{x}_{2}+\mu_{k} x_{2}}{1+\mu_{m}}-\left(x_{1}-x_{2}\right)$

The mass M1 rests on the mass M2 surface until $\left|f_{0}\right|>f_{s}$, and there is on impact because of dry friction.

When the mass M1 slips on the mass M2 surface, the impact occurs with $x_{1}(\mathrm{t})-x_{2}(\mathrm{t})=\mathrm{b}$. The conditions of conservation of momentum and the coefficient of restitution during the impact can be written as:

$\left\{\begin{array}{l}\dot{x}_{1+}+\mu_{m} \dot{x}_{2+}=\dot{x}_{1-}+\mu_{m} \dot{x}_{2-} \\ \dot{x}_{1+}-\dot{x}_{2+}=-r\left(\dot{x}_{1-}-\dot{x}_{2-}\right)\end{array} x_{1}-x_{2}=b\right.$

Where the subscript minus sign denotes the states just before the impact and the subscript plus sign denotes the states just after the impact, and $r$ is the coefficient of restitution.

Impacting systems are conveniently studied by using a map derived from the equations of motion. Each iterate of the map corresponds to the mass $M_{1}$ striking the mass $M_{2}$ once. Periodic-impact motions of the system are characterized by the symbol $n-p$, where $p$ denotes the number of impacts and $\mathrm{n}$ denotes the number of excitation periods. Periodic non-sticking motion can be defined as n-p-I in which two masses do not move in step during $\mathrm{n}$ excitation periods and there is no sticking motion. n-p-II represents another type of motion: during $n$ excitation periods, when the velocity of the mass $M_{1}$ becomes equal the mass $M_{2}$, the mass $M_{1}$ sticks to the mass $M_{2}$ until the static friction force overcomes maximum static friction, and the system enters into the slip mode once again. As a Poincare' section associated with the state of the vibro-impact system, just immediately before impact, the Poincare' section was selected

$$
\sigma=\left\{\left(x_{1}, \dot{x}_{1}, x_{2}, \dot{x}_{2}, \theta\right) \in R^{4} \times S, x_{1}-x_{2}=b, \dot{x}_{1}=\dot{x}_{1-}, \dot{x}_{2}=\dot{x}_{2-}\right\}
$$

to establish Poincare' map of the vibro-impact system with dry friction. 


\section{DYNAMIC ANALYSIS}

Numerical analyses were carried out for determining the dynamic responses of the vibro-impact system with dry friction. Due to the fact that the investigated system had dry friction, the system exhibited periodic motion with slip and stick phases. The non-dimensional parameters were assumed to have the following values

$\mu_{m}=10, \mu_{k}=5, \xi=0.1, b=0, f_{20}=0, f_{s}=0.15, R=0.08$.

The forcing frequency $\omega$ was taken as a control parameter in the dynamic analysis. Bifurcation diagrams, phase plane portraits and time series were plotted to illustrate the influence of the parametric excitation on the dynamic behavior of the system. The bifurcation diagrams of the system are shown in Fig. (9), in which the quantity $\omega t_{f} / 2 \pi$ and the relative velocity $\dot{x}_{2-}-\dot{x}_{1-}$ immediately before the impact are plotted versus the varying forcing frequency, where $t_{f}$ denotes the time of sliding motion of two masses between successive impacts. By examining the bifurcation diagrams shown in Fig. (2), large windows of periodic n-1 motion were observed for $\omega \in[0.56,10]$, with narrow areas of chaotic motion between two adjacent $\mathrm{n}-1$ motions. In the forcing frequency range interval $\omega \in[1.12,2.84]$, an impact was observed during the cycle of forcing. Between two consecutive impacts, non-stick motions occupied the majority of the parametric range and only in the forcing frequency range interval $\omega \in[1.47,1.6]$, slip-stick motions were observed. Considering an example of a period-1 sticking motion with forcing frequency of $\omega=1.5$. A time series of this periodic

(a)

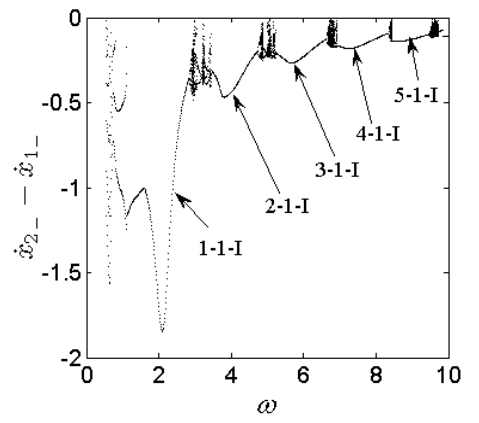

(b)

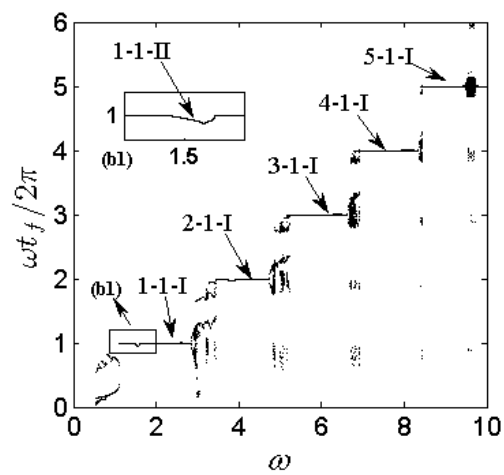

Fig. (2). Bifurcation diagrams $\left(f_{s}=0.15\right)$. motion is shown in Fig. (3a), and a Phase portrait in Fig. (3b). Stable 1-1-I motion was observed in the frequency interval $\omega \in[1.12,1.47] \&[1.6,2.84]$, as shown in Fig. (2). A Phase portrait of 1-1-I motion is shown for $\omega=2.2$ in Fig. (4b), and a time series in Fig. (4a). More interestingly, in the forcing frequency range interval $\omega \in[0.55,1.12]$, the chatter was observed to be induced by dry friction. As shown in Fig. (5) for $\omega=0.552$, when the timings between consecutive impacts became shorter until leading to stick model, and the relative velocities of two mass also became smaller until reaching zero in one periodic of chatter.

(a)

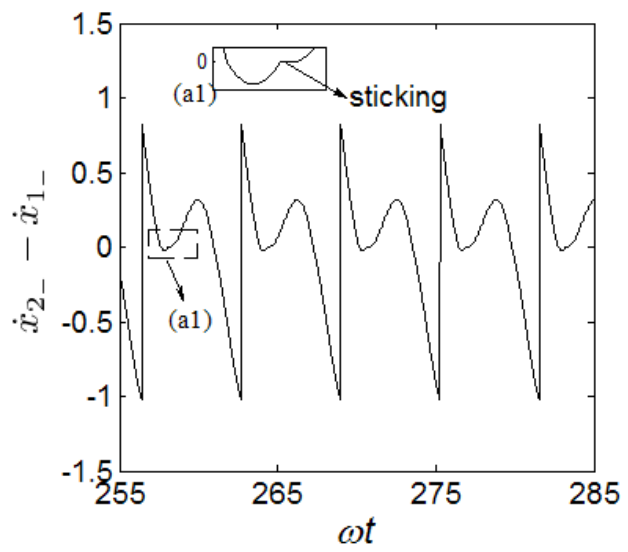

(b)

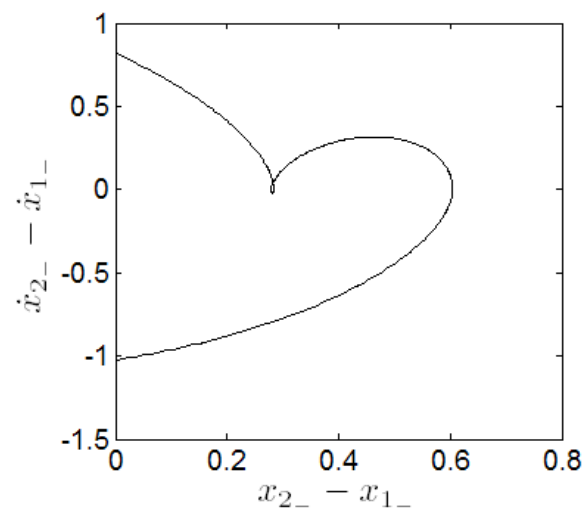

Fig. (3). Phase plane portrait and time series of relative motion, 11 -II motions $\omega=1.5$.

An observation of interest is the unusual route to chaos. A transition to chaos for the forcing frequency range interval $\omega \in[2.83,6.7]$ of the system with Coulomb friction can be observed in the bifurcation diagram as shown in Fig. (6). The transition from 1-1-I motion into chaos via double periodic bifurcation can be seen in Fig. (6a). With increased forcing frequency $\omega, 2-1-\mathrm{I}$ motion took place, and 4-2- I motion was stabilized (see Fig. 6b). The 4-2-I motion is represented by two fixed points in the projection of Poincare map, as shown in Fig. (7a, b). Instability of 4-2-I led to Hopf bifurcation of the motion, as a result the system exhibited quasi-periodic impact motion associated with 4-2-I motion. The quasi-periodic impact motion is represented by two attracting invariant circles in the projection of Poincare' map, as shown in Fig. (7c). Further increase in $\omega$, led 
(a)

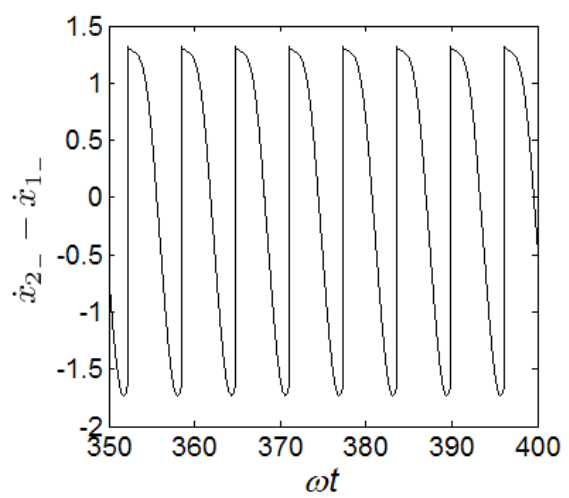

(b)

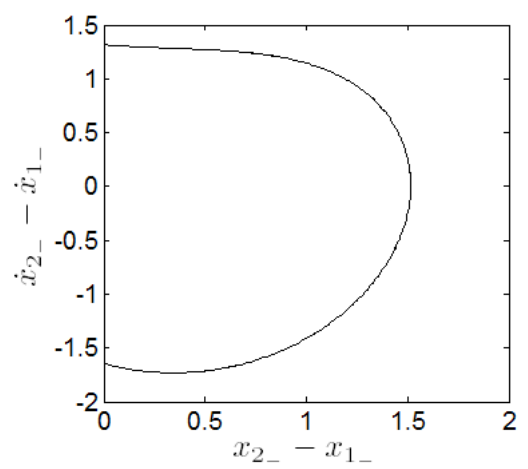

Fig. (4). Phase plane portrait and time series of relative motion, 11 -I motions $\omega=2$.

(a)

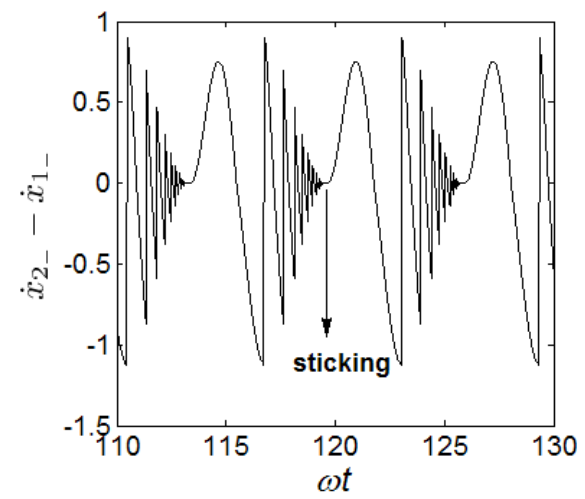

(b)

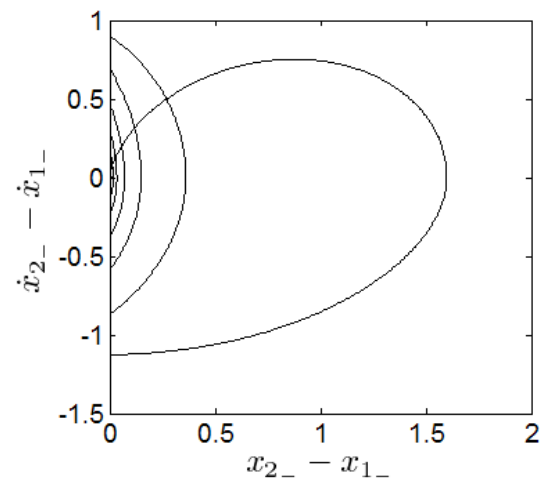

Fig. (5). Phase plane portrait and time series of relative motion, chatter $\omega=0.552$. to change in quasi-periodic motion into multiple periodic motions and into chaos via double periodic bifurcation followed. By analyzing the bifurcation diagrams shown in Fig. (6c), it was observed that 3-1- I motion changed s into 6-2- I motion via pitchfork bifurcation, and 6-2- I motion led into Hopf bifurcation of the motion, as a result of which subsequently unstable and chaotic friction force increased. Moreover, the bifurcation point shifted to the left by the influence of the dry friction in the higher frequencies.

(a)

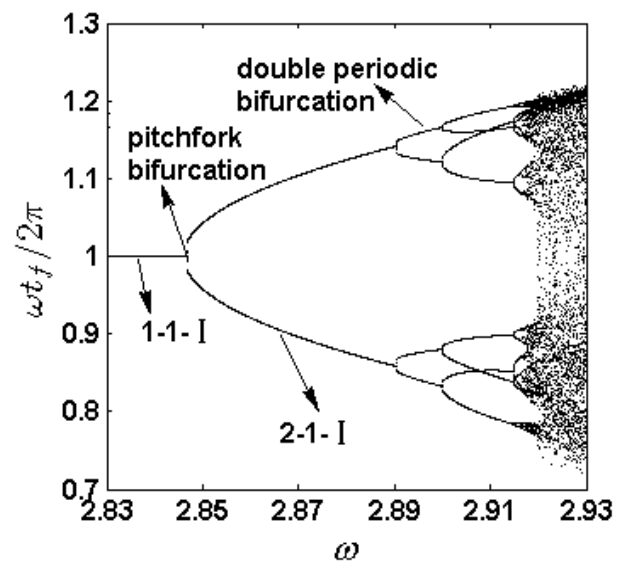

(b)

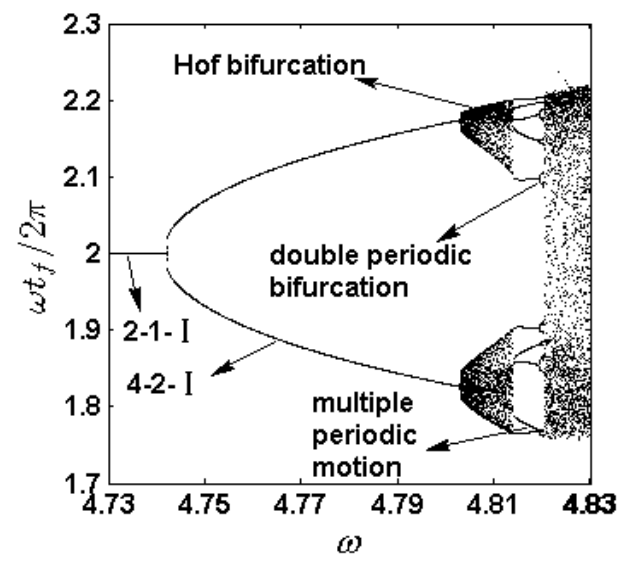

(c)

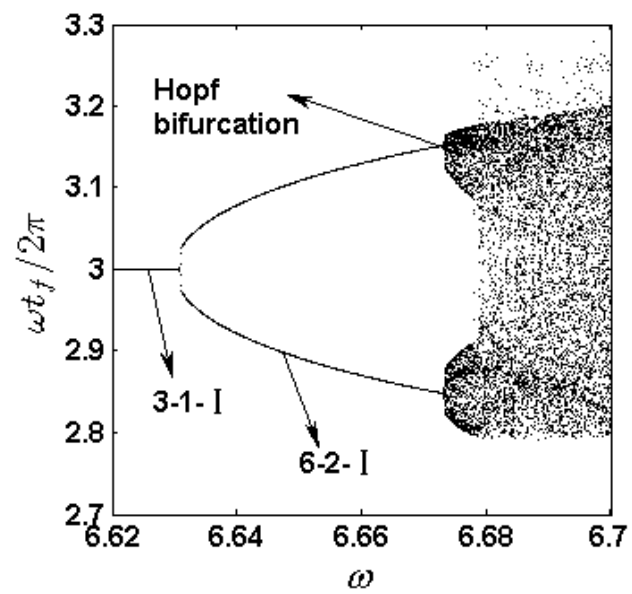

Fig. (6). Different routs of n-1-I motions leading to chaos $\left(f_{s}=0.15\right)$. 
(a)

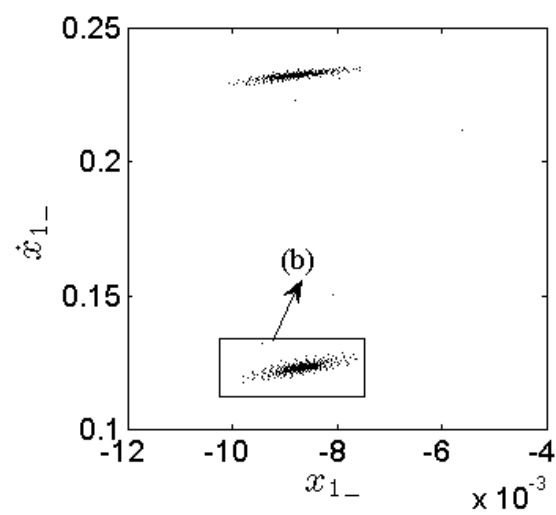

(b)

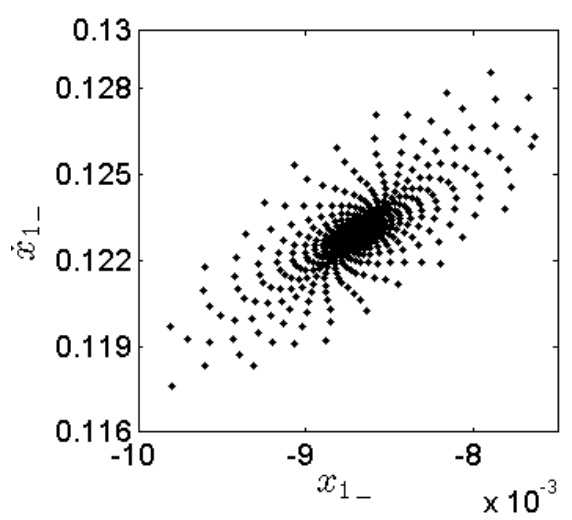

Fig. (7). Projected Poincaré section (a) and (b) 4-2- I fixed points, $\omega=4.801$; (b) attracting invariant circles associated with 4-2- I fixed points, $\omega=4.805$.

Owing to the existence of the non-smooth factors with friction and clearance, the dynamic behavior of the vibration system becomes complex. The bifurcation diagrams have been plotted to illustrate the influence of the dry friction on the dynamic behavior of the system.

As shown in Fig. (8), the influence of the dry friction is not obvious in the lower excitation frequencies, only the velocity of impact becomes smaller with increased friction. But in higher frequencies, less periodic motions take place whereas the chaotic motion area is broadened as shown in the figure.

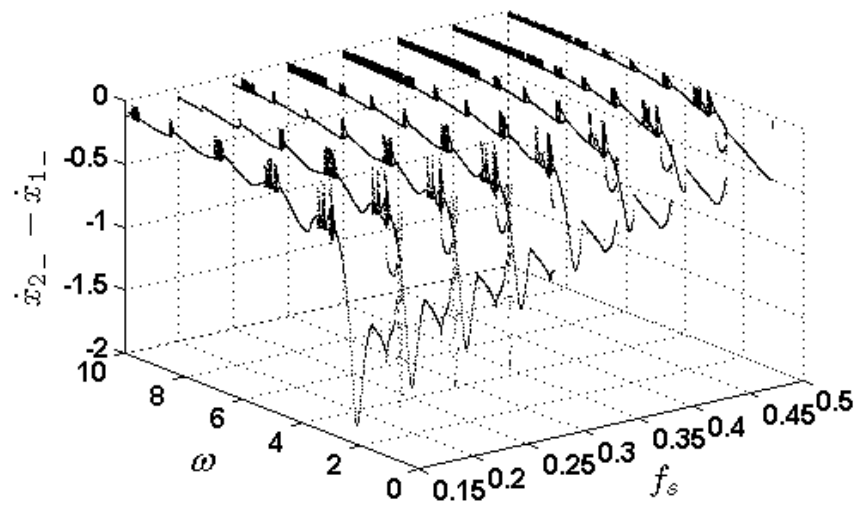

Fig. (8). Bifurcation diagram under different dry friction forces.

\section{CONCLUSION}

In this paper, the dynamics of two-degree-of-freedom vibro-impact system with dry friction has been studied. Numerical simulations helped determining the following conclusions.

(1) The system is characterized by sticking-periodic, non-sticking-periodic, quasi-periodic and chaotic attractors. The system exhibits sticking motions but they are exhibited in small amounts in the frequency range, and with the increase in friction, the proportion goes up. Furthermore, the chatter is also observed induced by dry friction in low frequency.

Different routes of n-1-I motions to chaos were also examined, and The Hof and period-doubling bifurcation for this oscillator were observed to be the main routs leading to chaos.

Fig. (8) shows the influence of the dry friction on the dynamic behavior of the system. With increased friction, the influence of the dry friction is not obvious in the lower excitation frequencies, only the velocity of impact becomes smaller with the friction increases. In higher frequencies, less periodic motions were observed whereas the windows of chaotic motion was broadened, and non-sticking periodic motions changed into sticking periodic motions gradually.

\section{CONFLICT OF INTEREST}

The authors confirm that this article content has no conflict of interest.

\section{ACKNOWLEDGEMENTS}

This work was supported by natural fund of GanSu province (Grant No. 1212RJZA044, 1208RJZA147 and 1212RJZA041), and The Young Scholars Science Foundation of Lanzhou Jiaotong University (Grant 2012039)

\section{REFERENCES}

[1] F. H. Yang, "Sliding bifurcations and chaos induced by dry friction in a braking system", Chaos, Solitons \& Fractals, vol. 8, pp. 079094, 2007.

[2] B. F. Feeny, and F. C. Moon, "Chaos in a forced dry-friction oscillator: Experiments and numerical modeling", Journal of Sound and Vibration, vol. 170, pp. 303-323, 1994.

[3] U. Galvanetto, and P. R. S. Bisho, "Characterization of the dynamics of a four-dimensional stick- slip system by a scalar variable", Chaos, Solitons \& Fractals, vol. 5, pp. 2171-2179, 1995.

[4] U. Galvanetto, "Some discontinuous bifurcations in a Two-block stick-slip system", Journal of Sound and Vibration, vol. 248, pp. 653-669, 2001.

[5] J. Awrejcewicz, "Holicke MM. Melnikov's method and stick-slip chaotic oscillations in very weakly forced mechanical applied scientists", Journal Bifurcation Chaos, vol. 9, pp. 505-518, 1999.

[6] J. Awrejcewicz, and D. Sendkowski, "How to predict stick-slip chaos in R4", Physics Letters A, vol. 330, pp. 371-376, 2004.

[7] J. Awrejcewicz, and D. Sendkowski, "Stick-slip chaos detection in coupled oscillators with friction", International Journal of Solids and Structures, vol. 42, pp. 5669-5682, 2005.

[8] J. J. Thmose, and A. Fidlin, "Analytical approximation for stickslip vibration amplitudes", International Journal of Non-linear Mechanics, vol. 38, pp. 389-403, 2003. 
[9] F. Peterka, "Analysis of Motion of the Impact-dry-friction Pair of Bodies and its Application to the Investigation of the Impact Dampers Dynamics", In: Proceedings of the ASME Design Technical Conference, Las-Vegas, 1999, pp. 12-15.
[10] K. M. Cone, and R. I. Zadoks, "A numerical study of an impact oscillator with addition of dry friction", Journal of Sound and Vibration, vol. 188, pp. 659-683, 1995.

Received: July 25, 2014

(C) Gao and Cao; Licensee Bentham Open.

This is an open access article licensed under the terms of the Creative Commons Attribution Non-Commercial License (http://creativecommons.org/licenses/by-nc/4.0/) which permits unrestricted, non-commercial use, distribution and reproduction in any medium, provided the work is properly cited. 